\title{
Detecting selection using Extended Haplotype Homozygosity-based statistics on unphased or unpolarized data
}

\author{
Alexander Klassmann ${ }^{1}$ and mathieu gautier ${ }^{2}$ \\ ${ }^{1}$ University of Cologne \\ ${ }^{2}$ INRAE
}

October 30, 2020

\begin{abstract}
Analysis of population genetic data often includes the search for genomic regions with signs of recent positive selection. One of the approaches involves the concept of Extended Haplotype Homozygosity (EHH) and its associated statistics. These statistics typically need phased haplotypes and, some of them, polarized variants. Here, we unify and extend previously proposed modifications to loosen these requirements. We compare the modified versions with the original ones by measuring the False Discovery Rate on simulated whole-genome scans and quantifying the overlap of inferred candidate regions on empirical data. We find that phasing information is indispensable for the accurate estimation of within-population statistics for all but very large samples and of cross-population statistics for small samples. Ancestry information, in contrast, is of lesser importance for both. Our publicly available $\mathrm{R}$ package rehh incorporates the modified statistics presented here.
\end{abstract}

\section{Hosted file}

rehh3_v14.pdf available at https://authorea.com/users/308075/articles/489834-detectingselection-using-extended-haplotype-homozygosity-based-statistics-on-unphased-or-

unpolarized-data 